\title{
Finding the Most Similar Concepts in two Different Ontologies
}

\author{
Adolfo Guzman-Arenas, Jesus M. Olivares-Ceja \\ Centro de Investigación en Computación, Instituto Politécnico Nacional \\ 07738 Mexico City, MEXICO \\ $\{$ a.guzman, jesuso\} @acm.org
}

\begin{abstract}
A concise manner to send information from agent A to B is to use phrases constructed with the concepts of A: to use the concepts as the atomic tokens to be transmitted. Unfortunately, tokens from A are not understood by (they do not map into) the ontology of B, since in general each ontology has its own address space. Instead, A and B need to use a common communication language, such as English: the transmission tokens are English words.

An algorithm is presented that finds the concept $\mathrm{C}_{\mathrm{B}}$ in $\mathrm{O}_{\mathrm{B}}$ (the ontology of B) most closely resembling a given concept $C_{A}$. That is, given a concept from ontology $\mathrm{O}_{\mathrm{A}}$, a method is provided to find the most similar concept in $\mathrm{O}_{\mathrm{B}}$, as well as the similarity sim between both concepts. Examples are given.
\end{abstract}

\section{Introduction and objectives}

How can we communicate our concepts, what we really mean? Two persons (or agents) A and B can communicate through previously agreed stereotypes, such as the calling sequence between a caller program and a called subroutine. This requires previous agreement between $\mathrm{A}$ and $\mathrm{B}$. This paper deals with communication with little previous consensus: $\mathrm{A}$ and $\mathrm{B}$ agree only to share a given communication language. The purpose of the communication is for $\mathrm{A}$ and for $\mathrm{B}$ to fulfill its objectives or goals. That is, we shall define a successful communication if A and B are closer to their goals as the result of such communication.

What can an agent do to meaningfully communicate with other agents (or persons), even when they had not made any very specific comitment to share a private ontology and communication protocol? Concept communication can not be fulfilled through direct exchange of concepts belonging to an ontology, since they do not share the same ontology. Instead, communication should be sought through a common language. Lucky agents can agree on a language whose words have a unique meaning. Others need to use an ambiguous language (such as a natural language) to share knowledge. This gives rise to imperfect understanding and confusion. This is the trust of this paper.

The objective of this work is to find the most similar (in meaning) object in B's ontology corresponding to a given object in A's ontology, and to measure their 
similarity. Example: Assume A wants to transmit its concept grapefruit ${ }^{1}$ to B. To this end, A translates it into word grapefruit, which is then transmitted to B. But B has no such word in its ontology. Thus, B asks A "what is a grapefruit?" A answers "it is a citric" (by seeing that citric is the father of grapefruit in $\mathrm{O}_{\mathrm{A}}$ ). Unfortunately, B has no concept to map word "citric". So B asks A "what is a citric?" $A$ answers "it is a fruit". Now, $O_{B}$ has concept fruit denoted by word fruit. But fruit $_{B}$ (the subindex B means "in $\mathrm{O}_{B}$ ") has several children: $B$ knows several fruits. Now $B$ has to determine wich of the children of fruit $_{B}$ most resembles grapefruit $t_{A}$. It may do so by seeing which child of fruit $_{B}$ has children quite similar to those children of grapefruit ${ }_{A}$. Or by seeing which fruits in $\mathrm{O}_{\mathrm{B}}$ have skin, bone, weight... similar to those of grape ruit $_{\mathrm{A}}$. Unfortunately, the problem is recursive: what is skin for $\mathrm{B}$ is epidermis for $\mathrm{A}$, and peel for $\mathrm{C}$. weight $\mathrm{A}_{\mathrm{A}}$ is in kilograms, whereas weight $t_{B}$ is in pounds. So the comparison has to continue recursively. §2 gives a precise description of the algorithm.



Figure 1. An ontology consists of a tree of concepts (nodes) under the subset relation (solid arrows), with other relations such as eats (dotted arrows), and with words associated to each concept (in parenthesis after each concept; some are omitted). Nodes also have (property, value) pairs, not shown in the figure

\subsection{Ontologies}

Knowledge is the concrete internalization of facts, attributes and relations among realworld entities - It is stored as concepts; it is measured in "number of concepts."

Concept. An object, relation, property, action, idea, entity or thing that is well known to many people, so that it has a name: a word(s) in a natural language. Examples: cat-chien, to_fly_in_air, angry_mad. So, concepts have names: those words used to denote them. A concept is unambiguous, by definition. Unfortunately, the names given by different people to a concept differ and, more unfortunately, the same word is given to two concepts (examples: words mole;

\footnotetext{
${ }^{1}$ In this paper, concepts appear in Courier font.
} 
star; can). Thus, words are ambiguous, ${ }^{2}$ while concepts are not. A person or agent, when receiving words from some speaker, has to solve their ambiguity in order to understand the speaker, by mapping the words to the "right" concept in his/her/its own ontology. The mapping of words to concepts is called disambiguation.

If two agents do not share a concept, at least partially, they can not communicate it or about it. A concept has (property, value) pairs associated with it.

Ontology. It is a formal explicit specification of a shared conceptualization [5]. $\bullet$ It is a hierarchy or taxonomy of the concepts we know. ${ }^{3}$ We represent an ontology as a tree, where each node is a concept with directed arcs (representing the relation subset and, at the leaves, perhaps the relation member_of instead of subset) to other concepts. Other relations (such as part_of, eats-ingests, 1ives_in, ...) can be drawn, with arcs of different types (figure 1). In general, these relations are also nodes in another part of the ontology.

Associated words. To each concept (node) there are several English words ${ }^{4}$ associated: those who denote it or have such concept as its meaning. Example: concept mad_angry has associated (is denoted by) words angry, crossed, pissedof, mad, irritated, incensed. Example: Word mole denotes a small_rodent, a spy_infiltrator and also a blemish_in_skin.

\subsection{Related work}

[12] represents concepts in a simpler format, called a hierarchy. Most works (for instance [11]) on ontologies involve the construction of a single ontology, even those that do collaborative design [8]. Often, ontologies are built for man-machine interaction [10] and not for machine-machine interaction. [1] tries to identify conceptually similar documents, but uses a single ontology. [3, 4] do the same using a topic hierarchy: a kind of ontology. [9] seeks to communicate several agents sharing a single ontology. The authors have been motivated $[6,7]$ by the need of agents to communicate with unknown agents, so that not much a priori agreement between them is possible. With respect to concept comparison, an ancestor of our COM $(\S 2$, appears first in [13]) matching mechanism is [2], based on the theory of analogy.

\section{Most similar concepts in two different ontologies}

The most similar concept $\mathrm{C}_{\mathrm{B}}$ in $\mathrm{O}_{\mathrm{B}}$ to concept $\mathrm{C}_{\mathrm{A}}$ in $\mathrm{O}_{\mathrm{A}}$ is found by the COM algorithm using the function $\operatorname{sim}\left(\mathrm{C}_{\mathrm{A}}\right)$ (called "hallar $\left(\mathrm{C}_{\mathrm{A}}\right)$ " in [13]) as described in the four cases below. It considers a concept, its parents and sons. In this section, for each case, a tree structure shows the situation and a snapshot of a screen presents an example. Assume that agent A emits (sends) to $\mathrm{B}$ words ${ }^{5}$ corresponding to $\mathrm{C}_{\mathrm{A}}$, and

\footnotetext{
${ }^{2}$ Some symbols or words are unambiguous: 3 , Abraham Lincoln, $\pi,\left(30^{\circ} \mathrm{N}, 15^{\circ} \mathrm{W}\right)$.

${ }^{3}$ Each concept that I know and has a name is shared, since it was named by somebody else.

${ }^{4}$ Or word phrases, such as "domestic animal".

${ }^{5}$ Remember, an agent can not send a node to another agent, just words denoting it.
} 
also sends words corresponding to the father of $\mathrm{C}_{\mathrm{A}}$, denoted by $\mathrm{p}_{\mathrm{A}}$. COM finds $\mathrm{C}_{\mathrm{B}}=$ $\operatorname{sim}\left(\mathrm{C}_{\mathrm{A}}\right)$, the concept in $\mathrm{O}_{\mathrm{B}}$ most similar to $\mathrm{C}_{\mathrm{A}}$. $\operatorname{sim}$ also returns a similarity value $s v$, a number between 0 and 1 denoting how similar such returned concept $C_{B}$ was to $C_{A}$.



Figure 2. Case (a). Words from $\mathrm{C}_{\mathrm{A}}$ and $\mathrm{p}_{\mathrm{A}}$ match words from $\mathrm{c}_{\mathrm{B}}$ and $\mathrm{p}_{\mathrm{B}}$



Figure 3. Case (b). Words from $p_{A}$ match words from $p_{B}$ but $c_{A}$ has no equivalence

Case a) We look in $\mathrm{O}_{B}$ for two nodes $\mathrm{p}_{B}$ and $\mathrm{C}_{\mathrm{B}}$, such that: (1) the words associated to $C_{B}$ coincide with most of the words (received by B from A) ${ }^{6}$ of $C_{A}$; and (2) the words associated to $\mathrm{p}_{\mathrm{B}}$ coincide with most of the words $^{6}$ corresponding to $\mathrm{p}_{\mathrm{A}}$; and (3) $p_{B}$ is the father, grandfather or great-grandfather ${ }^{7}$ of $c_{B}$.

If such $p_{B}$ and $C_{B}$ are found, then $C_{B}$ is the nearest concept to $C_{A}$; the answer is $C_{B}$ and the algorithm finishes returning $s v=1$. Figure 2 represents this situation. Figure 4 shows the screenshot of COM when seeking in B the concept most similar to apple $e_{\mathrm{A}}$. The answer is concept apple $\mathrm{e}_{\mathrm{B}}$ in B with $s v=1$.

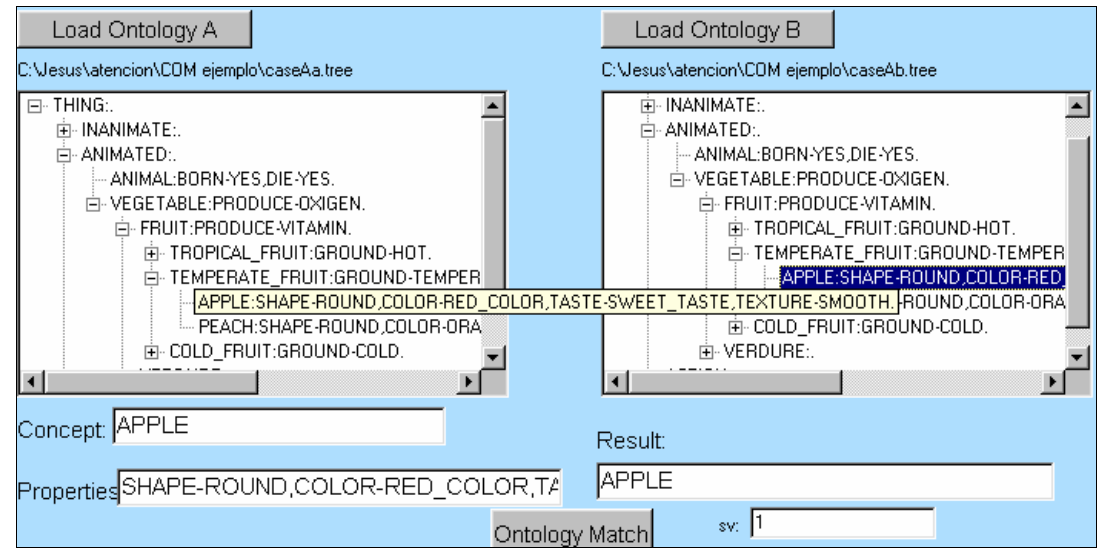

Figure 4. Case (a). Screen with the execution of COM for the case shown in Fig. 2

${ }^{6}$ We have found useful the threshold 0.5: more than half of the compared entities must coincide.

${ }^{7}$ If $\mathrm{p}_{\mathrm{B}}$ is found more than three levels up, the "semantic distance" is too high and sim says "no match." 
Case b) This case occurs when (2) of case (a) holds, but (1) and (3) do not. $\mathrm{p}_{B}$ is found in $\mathrm{O}_{\mathrm{B}}$ but $\mathrm{C}_{\mathrm{B}}$ is not. See Figure 3. In this case, sim (which Olivares calls hallar $)$ is called recursively, and we try to compute $\mathrm{p}_{\mathrm{B}}{ }^{\prime}=\operatorname{sim}\left(\mathrm{p}_{\mathrm{A}}\right)$ to confirm that $\mathrm{p}_{\mathrm{B}}$ is the ancestor of concept of interest $\left(\mathrm{C}_{\mathrm{A}}\right)$.

(1) If the $\mathrm{p}_{\mathrm{B}}$ ' found is thing, the root of $\mathrm{O}_{\mathrm{B}}$, the algorithm returns not_found and concludes; $s v=0$;

(2) Otherwise, a special child of $p_{B}$, to be called $c_{B}{ }^{\prime}$, is searched in $O_{B}$, such that:

A. Most $^{6}$ of the pairs (property, value) of $C_{B}{ }^{\prime}$ coincide with the corresponding pairs of $\mathrm{C}_{\mathrm{A}}$. Children of $\mathrm{p}_{\mathrm{B}}$ with just a few matching properties ${ }^{6}$ or values are rejected. If the candidate $C_{B}{ }^{\prime}$ analyzed has children, they are checked (using sim recursively) for a reasonable match ${ }^{6}$ with the children of $C_{A}$. If a $C_{B}{ }^{\prime}$ is found with the desired properties, the algorithm reports success returning $C_{B}{ }^{\prime}$ as the concept in $\mathrm{O}_{B}$ most similar to $\mathrm{C}_{\mathrm{A}} . S v=$ the fraction of pairs of $\mathrm{C}_{\mathrm{B}}{ }^{\prime}$ coinciding with corresponding pairs of $\mathrm{C}_{\mathrm{A}}$.

B. Otherwise $C_{B}$ ' is sought among the sons of the father (in $B$ ) of $p_{B}$; that is, among the brothers of $\mathrm{p}_{\mathrm{B}}$; if necessary, among the sons of the sons of $\mathrm{p}_{\mathrm{B}}$; that is, among the grandsons of $\mathrm{p}_{\mathrm{B}}$. If found, the answer is $\mathrm{C}_{\mathrm{B}}{ }^{\prime} . s v=$ the $s v$ returned by $\mathrm{C}_{\mathrm{B}}{ }^{\prime}$ multiplied by 0.8 if $\mathrm{C}_{\mathrm{B}}{ }^{\prime}$ was found among the sons of $\mathrm{p}_{\mathrm{B}},{ }^{8}$ or by $0.8^{2}=0.64$ if found among the grandsons of $\mathrm{p}_{\mathrm{B}}$.

C. If such $C_{B}{ }^{\prime}$ is not found, then the node nearest to $C_{A}$ is some son of $p_{B}$, therefore sim returns the remark (son_of $\mathrm{p}_{\mathrm{B}}$ ) and the algorithm concludes. $s v=0.5$ (an arbitrary but reasonable value). For example, if A sends words that correspond to the pair $\left(c_{A}=k i w i, p_{A}=f r u i t\right)$, and $\mathrm{B}$ has the concept fruit but doesn't have the concept kiwi nor any similar fruit, in this case, the concept $\mathrm{kiwi}$ (of A) is translated by B into (son_of fruit), which means "some fruit I don't know" or "some fruit I do not have in my ontology."

Figure 5 shows the execution of COM for case (b)2(A). In this case concept $\mathrm{kiw} \mathrm{i}_{\mathrm{A}}$ has no equivalent in B. Here rare_fruit $t_{B}$ is chosen from B as the most similar concept because parents coincide and properties of $k i w i_{A}$ and rare_fruit $t_{B}$ are similar (that was calculated using COM recursively for each property-value). $s v=0.8$ because the exact equivalent concept in B was not found.

Case c) This case occurs when (1) of case (a) holds but (2) and (3) do not. See figure 6. $c_{B}$ is found but $p_{B}$ is not. We try to ascertain whether the grandfather (in $\mathrm{O}_{B}$ ) of $\mathrm{C}_{\mathrm{B}}$ has words that match ${ }^{6}$ those of $\mathrm{p}_{\mathrm{A}}$ (corresponding words that are equal exceed $50 \%$ ), or if the great-grandfather of $\mathrm{C}_{B}$ in $\mathrm{O}_{B}$ has such matching ${ }^{6}$ words.

(1) If that is the case, the concept in $\mathrm{O}_{B}$ more similar to $\mathrm{p}_{A}$ is the grandfather (or the great-grandfather) of $\mathrm{C}_{\mathrm{B}}$, and the algorithm finishes returning $\mathrm{C}_{\mathrm{B}} . s v=0.8$ for the grandfather case, and $0.8^{2}$ for the great-grandfather case.

\footnotetext{
${ }^{8}$ We have found that 0.8 allows for a fast decay as one moves up from father to grandfather and up.
} 
(2) Otherwise (parents do not match), we verify two conditions:

A. Most ${ }^{6}$ of the properties (and their corresponding values) of $C_{B}$ should coincide (using sim) with those of $\mathrm{C}_{\mathrm{A}}$; and

B. Most of the children of $\mathrm{C}_{\mathrm{A}}$ should coincide (using sim) with most ${ }^{6}$ of the children of $\mathrm{C}_{\mathrm{B}}$.

If the properties in (A) and the children in (B) coincide, the algorithm concludes with response $C_{B}$, although it did not find in $O_{B}$ the $p_{B}$ that corresponds to the concept $\mathrm{p}_{\mathrm{A}}$ in $\mathrm{O}_{\mathrm{A}}$. $s v=$ the fraction of properties and children of $\mathrm{C}_{\mathrm{B}}$ matching with corresponding entities of $\mathrm{C}_{\mathrm{A}}$.

(3) If even fewer properties and children are similar then response is (probably $C_{B}$ ) and the algorithm finishes. $s v$ is computed like in (2)B.

(4) If neither properties nor children are similar, response is not_found and the algorithm finishes. $s v=0$.

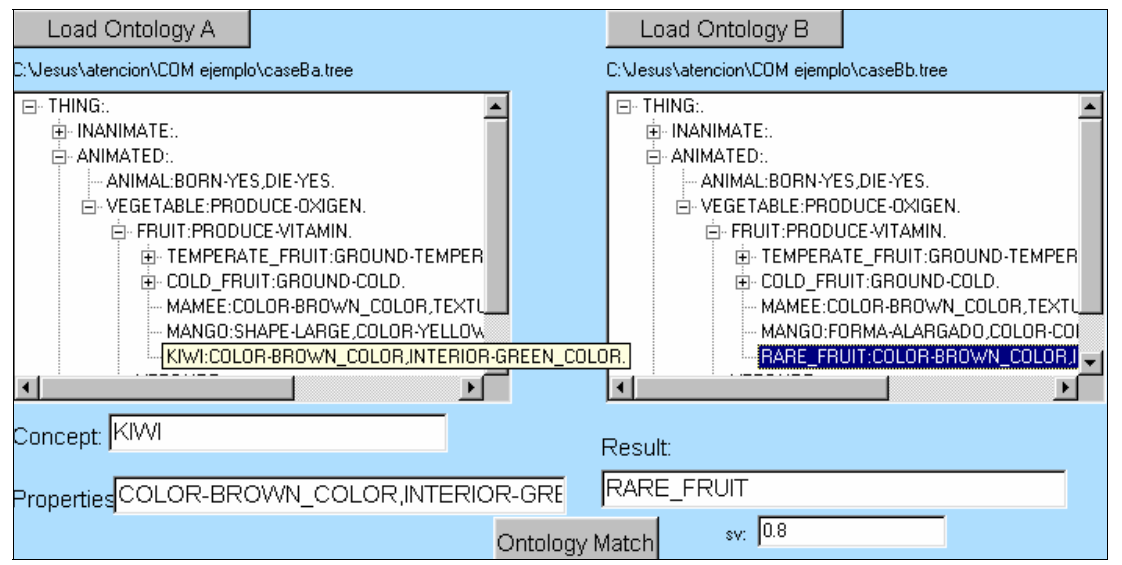

Figure 5. Case (b). Screen with the execution of COM corresponding to Figure 3

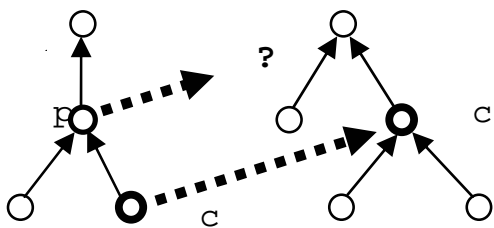

Figure 6. Case (c). Words from $\mathrm{C}_{\mathrm{A}}$ match with words of $C_{B}$ but there is no equivalence for words of $p_{A}$. See Figure 8

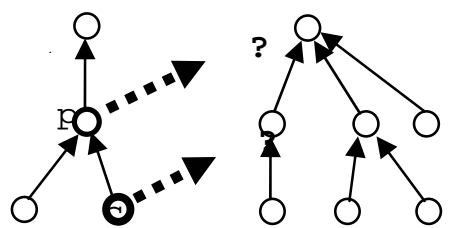

Figure 7. Case (d). There are no words from $\mathrm{c}_{\mathrm{A}}$ nor $\mathrm{p}_{\mathrm{A}}$ that match with words of $\mathrm{B}$

Figure 8 shows an example of case (c)(2). In this case we use COM to seek in B the most similar concept to apple $e_{\mathrm{A}}$. Here concepts match but parents do not (fruit $t_{\mathrm{A}}$, $f_{\circ o d_{B}}$ ) (words are different for each parent), therefore similarity of the properties are used (calling recursively to $\mathrm{COM}$ ). $s v=0.8$ because parents do not coincide. 




Figure 8. Case (c). Screen with the execution of COM corresponding to figure 6

Case d) If neither $c_{B}$ nor $p_{B}$ are found, the algorithm concludes returning the response not_found. $s v=0 . \mathrm{C}_{\mathrm{A}}$ could not find a similar node in $\mathrm{O}_{\mathrm{B}}$. The agents may have different ontologies (they know about different subjects) or they do not share a common communication language. See figures 7 and 9.

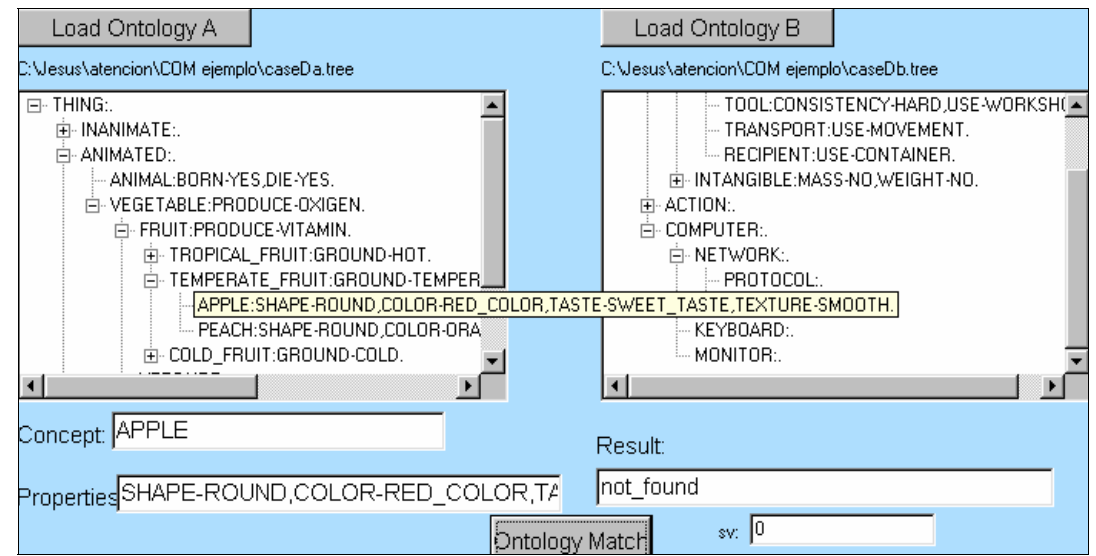

Figure 9. Case (d). Screen with the execution of COM for case (d). Ontologies refer mostly to different areas. COM returns not_found with $s v=0$

Figure 9 shows the execution of case (d). Observe that ontology $\mathrm{O}_{\mathrm{A}}$ is mainly about fruits while $\mathrm{O}_{B}$ is mainly about Computer Science. There are some concepts in common, but not the involved concepts. $s v=0$.

sim is not symmetric. If $\mathrm{C}_{\mathrm{B}}$ is the concept most similar to $\mathrm{C}_{\mathrm{A}}$, it is not necessarily true that $\mathrm{C}_{\mathrm{A}}$ is the concept most similar to $\mathrm{C}_{\mathrm{B}}$. Example: $\mathrm{O}_{\mathrm{A}}$ knows ten kinds of 
hamme $r_{A}$, while $\mathrm{O}_{B}$ only knows hammer $r_{B}$ (a general hammer). Then, COM maps each of the ten hammer $r_{A}$ into hammer $r_{B}$, while hammer $r_{B}$ best maps into, say, hammer_for_carpenter ${ }_{\mathrm{A}}$ [12].

The function $\operatorname{sim}$ is only defined between a concept $\mathrm{C}_{\mathrm{A}}$ in $\mathrm{O}_{\mathrm{A}}$ and the most similar concept $\mathrm{C}_{\mathrm{B}}$ in $\mathrm{O}_{\mathrm{B}}$.

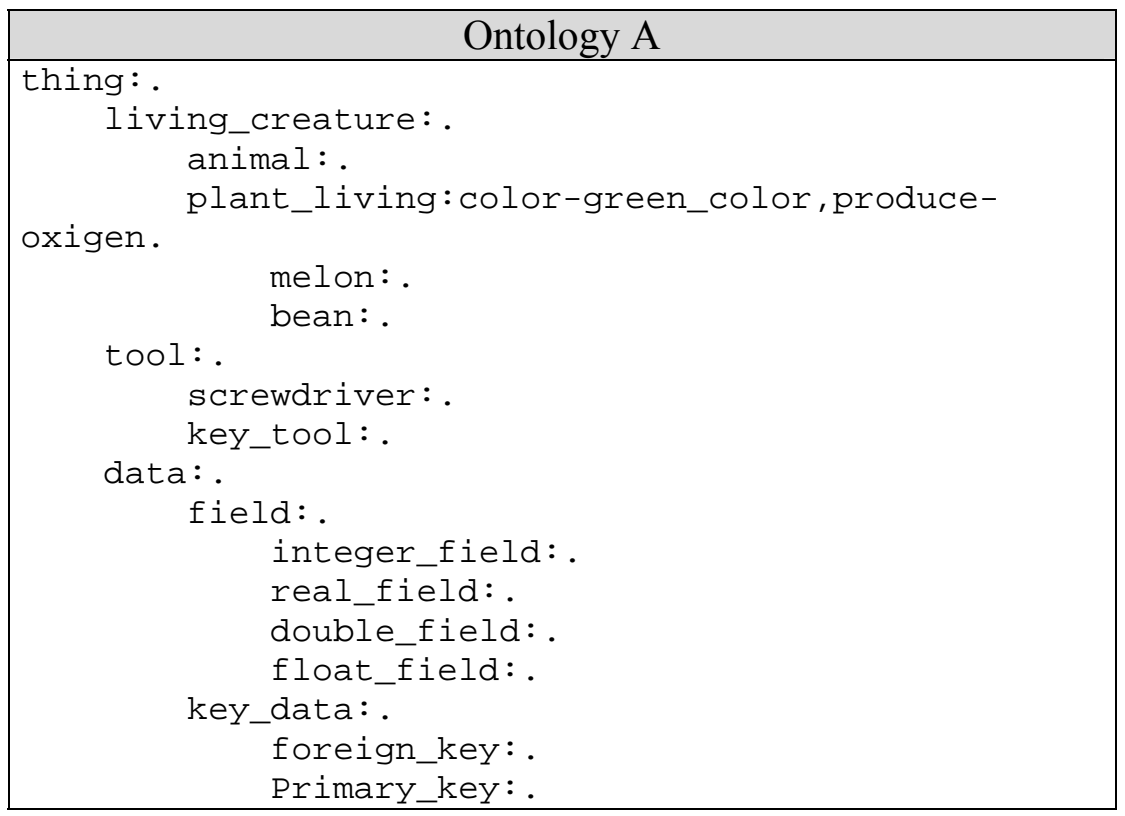

Figure 10. Ontology A. Used to compute similarity to concepts in ontology B

\subsection{Examples of similarity}

Now we give examples for sim, the similarity between two concepts, each from one ontology. Here we assume that properties like relations and colors are part of both ontologies. For simplicity properties are shown only where needed. Properties appear after the colon as relation-value pairs. For ontologies A and B (Figures 10 and 11):

$\operatorname{sim}\left(\mathrm{field} \mathrm{d}_{\mathrm{A}}\right)=\mathrm{field} \mathrm{d}_{\mathrm{B}}$ with $s v=1$ because words of concepts and parents coincide. This is an example of case (a).

$\operatorname{sim}\left(\mathrm{key}_{-} \mathrm{too} l_{\mathrm{A}}\right)=\mathrm{key} \_$tool $l_{\mathrm{B}}$ with $s v=1$. This is an example of case(a), where words of the parent and concept in A match words of corresponding nodes in B. Although word 'key' denotes (belongs to the associated words of) both concepts key_dat $a_{B}$ and key_tool $l_{B}$, the words of $t o \circ l_{A}$ only map into those of tool $l_{B}$ and key_tool ${ }_{B}$ is selected without ambiguity.

$\operatorname{sim}\left(\right.$ screwdriver $\left._{\mathrm{A}}\right)=\left(\right.$ son_of $\left.\operatorname{tool}_{\mathrm{B}}\right)$ with $s v=0.5$. This is case $(\mathrm{b})$ : parents coincide, but in ontology $\mathrm{B}$ there is no concept similar to screwdriver $\mathrm{A}_{\mathrm{A}}$, therefore the algorithm detects that agent $A$ is referring to a son of concept too $l_{B}$. 
$\operatorname{sim}\left(\right.$ plant_living $\left.\mathrm{A}_{\mathrm{A}}\right)=$ vegetable $_{\mathrm{B}}$ with $s v=0.8$. This an example of case (b) when parents coincide but the concepts do not. In this case properties of concepts are used to establish the similarity among concepts. The similarity of the properties is calculated using the COM recursively for each property and value.

$\operatorname{sim}\left(\right.$ double_field $\left.d_{\mathrm{A}}\right)=$ not_found and $s v=0$. This is an example of case (d) when no concept nor parent are found in B. The ontology A has sent B a concept of which B has no idea.

$\operatorname{sim}\left(\operatorname{melon}_{\mathrm{A}}\right)=$ not_found and $s v=0$. This is other example of case (d) where words sent to B from A do not match a pair parent-concept in B.

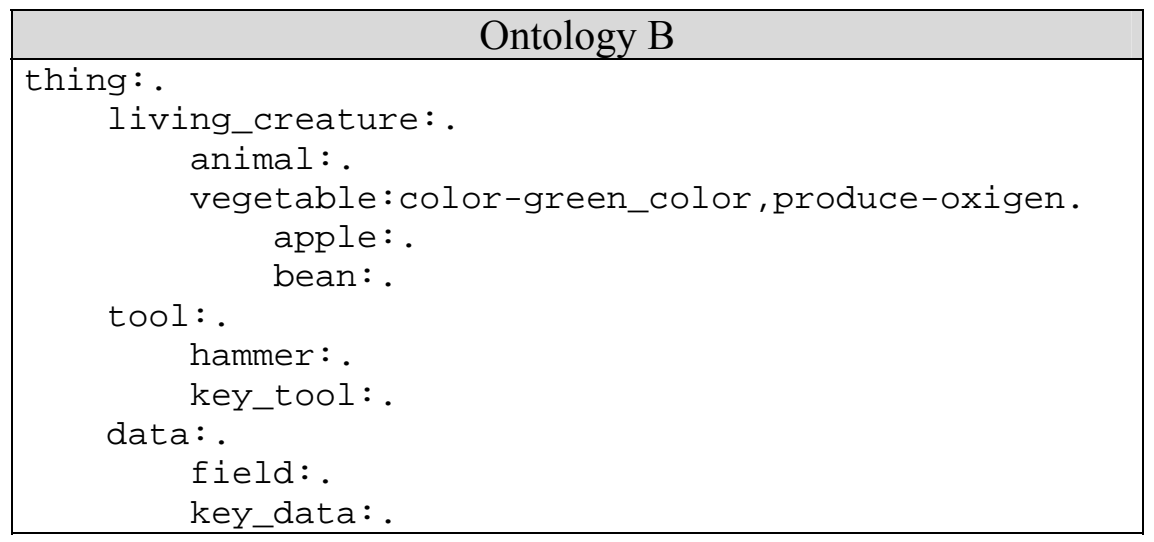

Figure 11. Ontology B. Used to compute similarity to concepts in ontology A

\subsection{Conclusions}

Methods embodied in a computer program are given to allow concept exchange and understanding between agents with different ontologies, so that there is no need to agree first on a standard set of concept definitions. Given a concept, a procedure for finding the most similar concept in another ontology is shown. The procedure also finds a measure of the similarity $s v$ between concepts $C_{A}$ and $C_{B}$. Our methods need further testing against large, vastly different, or practical ontologies.

In contrast, most efforts to communicate two agents take one of these approaches:

1. The same person or programmer writes (generates) both agents, so that preestablished ad hoc communicating sequences ("calling sequences," with predefined order of arguments and their meaning) are possible. This approach, of course, will fail if an agent is trying to communicate with agents built by somebody else.

2. Agents use a common or "standard" ontology to exchange information. This is the approach taken by CYC [11]. Standard ontologies are difficult and slow to build (they have to be designed by committee, most likely). Another deficiency: since new concepts appear each day, they slowly trickle to the standard ontology, so that it always stays behind current knowledge. 
Even for approach (2), a language to convey other entities built out of concepts: complex objects (which do not have a name), actions, desires, plans, algorithms... (not just concepts) is needed. Such language is beyond this paper; hints of it at [13].

Our approach allows communication in spite of different ontologies, and needs neither (1) nor (2).

Acknowledgments. Work herein reported was partially supported by NSFCONACYT Grant 32973-A and Project CGPI-IPN 18.07 (20010778). Olivares received a PIFI research assistantship from CGPI-IPN. Guzman-Arenas has a SNI National Scientist Award from SNI-CONACYT.

\section{References}

1. John Everett, D Bobrow, R Stolle, R Crouch, V de Paiva, C Condoravdi, M van den Berg, and L Polyani. (2002) Making ontologies work for resolving redundancies across documents. Communication of the ACM 45, 2, 55-60. February.

2. K. Forbus, B. Falkenhainer, D. Gentner. (1989) The structure mapping engine: algorithms and examples. Artificial Intelligence 41, 1, 1-63.

3. A. Gelbukh, G. Sidorov, and A. Guzman-Arenas. (1999) Use of a weighted document topic hierarchy for document classification. Text, Speech, Dialogue, 133-138. Pilsen, Chech Republic, September 13-17.

4. A. Gelbukh, G. Sidorov, and A. Guzman-Arenas. (1999) Document comparison with a weighted topic hierarchy. DEXA-99, 10th International Conference on Database and Expert System applications, Workshop on Document Analysis and Understanding for Document Databases, 566-570. Florence, Italy, August 30 to September 3.

5. Thomas R. Gruber (1993) Toward Principles for the Design of Ontologies Used for Knowledge Sharing, in Formal Ontology in Conceptual Analysis and Knowledge Representation, Nicola Guarino and Roberto Poli (eds.), Kluwer Academic Publishers.

6. A. Guzman, Jesus Olivares, Araceli Demetrio and Carmen Dominguez, (2000) Interaction of purposeful agents that use different ontologies. Lecture Notes in Artificial Intelligence (LNAI) 1793, 557-573. Osvaldo Cairo, Enrique Sucar, F. J. Cantu (eds). Springer Verlag.

7. A. Guzman, C. Dominguez, and J. Olivares. (2002) Reacting to unexpected events and communicating in spite of mixed ontologies In LNAI 2313, 377-386.

8. Cloyde W. Holsapple and K. D. Joshi. (2002) A collaborative approach to ontology design. Comm. ACM 45, 2, 42-47. February.

9. M. N. Huhns; M. P. Singh. and T. Ksiezyk (1997) Global Information Management Via Local Autonomous Agents. In Readings in Agents, M. N. Huhns, Munindar P. Singh, (eds.). Morgan Kauffmann Publishers, Inc. San Francisco, CA

10. Henry Kim. (2002) Predicting how ontologies for the semantic web will evolve. Comm. $A C M$ 45, 2, 48-54. February.

11. Douglas B. Lenat, R. V. Guha, Karen Pittman, Dexter Pratt and Mary Shepherd (1990) Cyc: Toward Programs with Common Sense, Comm. ACM 33, 9, $30-49$.

12. Serguei Levachkine, A. Guzman-Arenas (2002) Hierarchy as a new data type for qualitative variables. Submitted to Data and Knowledge Engineering.

13. Jesus Olivares (2002) An Interaction Model among Purposeful Agents, Mixed Ontologies and Unexpected Events. Ph. D. Thesis, CIC-IPN. In Spanish. Available on line at http:// www.jesusolivares.com/interaction/publica 